\title{
Balance potasio:calcio, relación con el déficit de presión de vapor y la radiación fotosintéticamente activa en tomate de invernadero Potassium:calcium balance, relationship with vapor pressure deficit and photosynthetically active radiation in greenhouse tomato
}

\author{
Ramiro Salas-Rivera $^{1}$ (i), Luis Alonso Valdez-Aguilar ${ }^{1:} \mathbb{C}^{\mathbb{D}}$, Daniela Alvarado-Camarillo ${ }^{1}$ (D), \\ Emilio Rascón-Alvarado ${ }^{2}$, Fidel Maximiano Peña-Ramos ${ }^{2}$ (i) yosé Antonio González-Fuentes $^{1}$ (i) \\ ${ }^{1}$ Departamento de Horticultura, ${ }^{2}$ Departamento de Ciencias del Suelo, Universidad Autónoma Agraria Antonio Narro. Calzada Antonio Narro 1923, Buenavista. \\ 25315 Saltillo, Coahuila, México. \\ *Autor para correspondencia (luisalonso.valdez@uaaan.edu.mx)
}

\section{RESUMEN}

La producción de tomate en invernadero demanda el conocimiento de factores técnicos como el manejo de la solución nutritiva y la interacción de ésta con las condiciones del ambiente. En la solución nutritiva pueden ocurrir desbalances entre nutrimentos como el potasio $(\mathrm{K})$ y calcio $(\mathrm{Ca})$; a su vez, el flujo de la savia y la translocación de éstos está influenciado por el por déficit de presión de vapor (DPV) y radiación fotosintéticamente activa (RFA). El presente estudio se realizó con los objetivos de determinar el balance óptimo de $\mathrm{K}$ y $\mathrm{Ca}$ en función del DPV y RFA prevalecientes durante el desarrollo de los frutos de los primeros 10 racimos en tomate; los primeros cinco racimos se desarrollaron durante la primavera, mientras que los racimos sexto al décimo durante el verano. Las plantas se irrigaron con soluciones nutritivas cuyos balances K:Ca fueron: 0.54, 0.64, 0.78, y 0.82. Los balances no afectaron calidad de frutos en primavera, aunque en verano, los balances 0.64 y 0.78 redujeron los sólidos solubles. En primavera, el rendimiento en cada uno de los cinco racimos estuvo influenciado por el balance K:Ca así como por el DPV; los racimos tuvieron un mayor rendimiento cuando el balance $\mathrm{K}: \mathrm{Ca}$ fue de 0.64 y el DPV fue de $2.81 \mathrm{KPa}$. Durante el verano, el rendimiento más alto fue en plantas tratadas con un balance de 0.54 y el DPV fue 2.50 o $2.74 \mathrm{KPa}$. $\mathrm{El}$ balance $\mathrm{K}: \mathrm{Ca}$ durante la primavera se correlacionó

Cita recomendada:

Salas-Rivera, R., L. A. Valdez-Aguilar, D. Alvarado-Camarillo, E. RascónAlvarado, F. M. Peña-Ramos y J. A. González-Fuentes. 2020. Balance potasio: calcio, relación con el déficit de presión de vapor y la radiación fotosintéticamente activa en tomate de invernadero. Terra Latinoamericana 38: 301-311.

DOI: https://doi.org/10.28940/terra.v38i2.589 negativamente con el DPV, sin embargo, en el verano, la correlación fue positiva. El balance $\mathrm{K}$ : Ca óptimo debe ser de 0.78-0.82 al subir la RFA hasta un máximo de $102-142 \mu \mathrm{mol} \mathrm{m}^{-2} \mathrm{~s}^{-1}$, mientras que niveles extremos de RFA deben ir acompañados de una reducción en el balance $\mathrm{K}$ :Ca.

Palabras clave: balance de cationes, humedad relativa, relaciones iónicas, temperatura.

\section{SUMMARY}

Greenhouse tomato production requires technical knowledge such as the management of the nutrient solution and its interaction with environmental conditions. Imbalances between nutrients such as potassium $(\mathrm{K})$ and calcium $(\mathrm{Ca})$ can occur in the nutrient solution; Moreover, the flow of sap and nutrients translocation are affected by the vapor pressure deficit (VPD) and photosynthetically active radiation (PAR). The present study was carried out with the objective to determine the optimal balance of $\mathrm{K}$ and $\mathrm{Ca}$ depending on VPD and PAR prevailing during the development of the fruits of 10 clusters in tomato; the first five clusters were developed during the spring, while the clusters sixth to tenth during the summer. Plants were irrigated with solutions whose $\mathrm{K}: \mathrm{Ca}$ balance was: $0.54,0.64,0.78$, and 0.82 . The balance did not affect fruit quality in spring, although in summer, balances

Recibido: 10 de julio de 2019 . Aceptado: 13 de febrero de 2020. Publicado en Terra Latinoamericana 38: 301-311. 
0.64 and 0.78 reduced soluble solids. In spring, fruit yield in each of the five clusters was influenced by the $\mathrm{K}$ :Ca balance as well as by the VPD; the clusters had a higher fruit production when the $\mathrm{K}$ :Ca balance was 0.64 and the VPD was $2.81 \mathrm{KPa}$. During the summer, the highest yield in fruits was in plants treated with a balance of 0.54 and the VPD was 2.50 or $2.74 \mathrm{KPa}$. The $\mathrm{K}$ :Ca balance during the spring correlated negatively with the VPD, however, in the summer, the correlation was positive. In spring, the $\mathrm{K}: \mathrm{Ca}$ optimum balance should be $0.78-0.82$ when PAR reached a maximum of 102-142 $\mu \mathrm{mol} \mathrm{m} \mathrm{m}^{-2} \mathrm{~s}^{-1}$, while extreme PAR levels should be accompanied by a reduction in the $\mathrm{K}$ :Ca balance.

Index words: cation balance, relative humidity, ionic relations, temperature.

\section{INTRODUCCIÓN}

México es un país en desarrollo que ha tomado un papel preponderante como proveedor de productos hortícolas en el mercado internacional (Van den Broeck y Martens, 2016). El cultivo del tomate (Solanum lycopersicon L.) es la actividad agroindustrial más importante por sus implicaciones sociológicas debido a la escala de producción así como por los ingresos económicos que genera (Barron y Rello, 2000). En un lapso de 21 años, la producción de tomate se incrementó en $125 \%$ en México desde la entrada en vigor de tratados comerciales con Estados Unidos y Canadá en 1994 (Soto, 2018), lo cual ha permitido que México sea el décimo país en la producción de este cultivo y el primero en exportaciones a nivel mundial (Saavedra, 2017; SIAP, 2018). Para lograr esto, los productores de esta especie debieron cumplir con altos estándares de calidad, sanidad e inocuidad.

El cultivo de tomate en invernadero permite un mejor control de parámetros ambientales y biológicos, lo que se refleja en un mayor rendimiento y calidad de frutos. Sin embargo, debido a inadecuadas prácticas de fertilización y nutrición, se han presentado problemas de deterioro del suelo en los invernaderos, como pérdida de propiedades físicas y químicas, mismas que merman la productividad. Bajo tal situación, la producción de tomate en invernadero en sistemas de cultivo sin suelo representa una alternativa viable.

En un sistema de producción de cultivos sin suelo se debe considerar el manejo de la nutrición mineral ya que este es un factor clave que determina el rendimiento y la calidad (Fanasca et al., 2006). Este manejo incluye la composición de la solución nutritiva (SN) (Villegas et al., 2005), la relación mutua entre cationes y aniones, la concentración de los nutrimentos, la conductividad eléctrica (CE), el pH, y la temperatura (Adams y Ho, 1995; Villegas et al., 2005).

Debido a que la absorción de los macronutrimentos no es lineal durante el desarrollo de la planta, la relación mutua entre los iones de la $\mathrm{SN}$ debe ajustarse periódicamente para evitar desbalances nutrimentales, como es el caso del potasio (K) y el calcio (Ca) (Lara, 1999; Schneider y Mollier, 2016).

El K es el elemento que más demandan los frutos de tomate (Betancourt y Pierre, 2013) y está implicado en la calidad del mismo (Fanasca et al., 2006). Una alta relación catiónica de $\mathrm{K}$ en la $\mathrm{SN}$, con respecto a $\mathrm{Ca}$, aumenta los atributos de calidad (Fanasca et al., 2006); ya que plantas de tomate irrigadas con altas concentraciones de $\mathrm{K}$, combinado con bajo $\mathrm{Ca}$, resulta en frutos con mayor concentración de licopeno (Almeselmani et al., 2009).

En cuanto al $\mathrm{Ca}$, un aporte adecuado de este elemento logra reducir el porcentaje de frutos afectados por pudrición apical, permitiendo aumentar la calidad comercial (Parra et al., 2008). Paiva et al. (1998) reportaron que a medida que aumenta la concentración de $\mathrm{Ca}$ en la solución que rodea las raíces, la absorción de $\mathrm{K}$ en el fruto disminuye, mientras que al aplicar una concentración baja de $\mathrm{K}$ con respecto al $\mathrm{Ca}$ se incrementa la absorción y transporte de Ca. Por lo tanto, un aporte desbalanceado de $\mathrm{K}$ y $\mathrm{Ca}$ en la $\mathrm{SN}$ puede disminuir la calidad y rendimiento (Lara, 1999), como han demostrado Nukaya et al. (1995).

El cultivo de tomate en invernaderos con ambiente semi controlado y de mediana tecnología, se prolonga por varias estaciones del año, lo que implica que las plantas están expuestas a condiciones ambientales contrastantes, como el déficit de presión de vapor (DPV) y la radiación fotosintéticamente activa (RFA). El DPV mide la deficiencia de humedad en la atmosfera, a una humedad relativa y temperatura determinadas, en comparación con la humedad que debería de tener si la atmósfera estuviera saturada. Este parámetro determina la tasa de transpiración ya que a un bajo DPV, es decir, una baja demanda de agua por la atmósfera, está relacionado con una baja tasa transpiratoria, lo que a su vez causa un reducido flujo 
de la savia en el tallo (Swaef y Steppe, 2010). Por lo tanto, el bajo o alto flujo de savia ocasionado por un bajo o alto DPV, respectivamente, pueden afectar el absorción y translocación de nutrimentos (Lara, 1999; Díaz et al., 2007; Suzuki et al., 2015).

Condiciones contrastantes de DPV se reflejan en cambios en la tasa transpiratoria, flujo de savia $\mathrm{y}$ transporte de nutrimentos. Bertin et al. (2000) reportaron que un bajo $\mathrm{DPV}$, menor de $1.5 \mathrm{KPa}$, atenuó la disminución en el peso fresco de plantas de tomate debido al aumento en el peso fresco del fruto; sin embargo, los autores también señalan que un bajo DPV reduce la incidencia de pudrición apical del fruto, aunque aumenta la presencia de rajaduras en el mismo. Tanto la pudrición apical como la rajadura del fruto están relacionadas con el $\mathrm{K}$ y Ca, por lo que el balance óptimo entre estos cationes está influenciado por el DPV.

Se ha demostrado en tomate que subir la RFA de 249 a $1089 \mathrm{~mol} \mathrm{~m}^{-2}$ está relacionado con un aumento en el peso seco, sólidos solubles totales y firmeza del fruto; sin embargo, un incremento en la temperatura acumulada estuvo relacionado con un aumento en, además de los anteriores parámetros, el contenido de jugo, $\mathrm{pH}, \mathrm{CE}$ y acidez titulable, por lo que se concluye que la calidad del fruto en tomate depende más de la temperatura acumulada que de la RFA (Riga et al., 2008).

Los objetivos de este trabajo fueron el determinar la relación óptima entre $\mathrm{K}$ y Ca en la $\mathrm{SN}$ y el efecto de esta sobre el rendimiento y calidad en tomate en un sistema de cultivo sin suelo en dos etapas de fructificación: primavera y verano, y determinar la relación entre el balance K:Ca con el DPV y la RFA prevalecientes en estas estaciones.

\section{MATERIALES Y MÉTODOS}

La presente investigación se desarrolló en un invernadero de tecnología media, el cual contaba con cubierta de plástico, sistema de enfriamiento por muro húmedo y extractor, calefacción y malla anti-insectos. El invernadero se encuentra ubicado en el Departamento de Horticultura de la Universidad Autónoma Agraria Antonio Narro en Saltillo, Coahuila, México (25 ${ }^{\circ} 21^{\prime}$ 24.37" N, $101^{\circ} 02^{\prime} 05.45^{\prime \prime}$ O, $1762 \mathrm{~m}$ de altitud).

Se utilizaron semillas de tomate variedad Clermon. El trasplante se efectuó el 8 de marzo de 2016 en contenedores de polietileno negro, los cuales se llenaron con $10 \mathrm{~L}$ de una mezcla de turba ácida (Sphagnum moss, PREMIERE, Canadá) y perlita (Hortiperl, Termolita Monterrey, México) en proporción de 70:30\% (v/v). Las plantas se establecieron en hileras dobles con una distancia de $1.2 \mathrm{~m}$ de centro a centro y a una distancia de $0.4 \mathrm{~m}$ entre planta y planta.

La cosecha de frutos se realizó en dos estaciones del año, primavera (del 5 de junio al 24 de julio de 2016) y verano (del 25 de julio al 5 de septiembre de 2016). Se hizo un aclareo a 5 frutos por racimo en todas las plantas y la cosecha inició a los 82 días después del trasplante. En ambas estaciones del año se evaluaron los efectos de los balances K:Ca en la SN $(0.54,0.64,0.78$, y 0.82) (Cuadro 1). Se consideraron las propiedades químicas del agua de riego para elaborar las $\mathrm{SN}$ y se ajustó el $\mathrm{pH}$ en un rango de 5.5 a 6.0 .

Las SN Se aplicaron mediante un sistema de riego por goteo con dos goteros por planta cuyo gasto era de $4 \mathrm{~L} \mathrm{~h}^{-1}$ cada uno.

Los frutos de los racimos 1 al 5 comprendieron a los producidos durante la primavera mientras que los del racimo 6 al 10 a los producidos durante el verano. En

Cuadro 1. Concentración de macronutrimentos en soluciones nutritivas y balance K:Ca aplicadas en dos estaciones del año: primavera y verano.

Table 1. Macronutrient concentration in nutritional solutions and K:Ca balance applied in two seasons of the year: spring and summer.

\begin{tabular}{lccccccc}
\hline Balance K:Ca & $\mathrm{NO}_{3}^{-}$ & $\mathrm{H}_{2} \mathrm{PO}_{4}^{-}$ & $\mathrm{SO}_{4}^{2-}$ & $\mathrm{K}^{+}$ & $\mathrm{Mg}^{2+}$ & $\mathrm{Ca}^{2+}$ & $\mathrm{Conductividad} \mathrm{eléctrica}^{2+}$ \\
\hline & $-\ldots$ & 2 & 8 & 7 & 4 & 13 & 2.4 \\
0.54 & 14 & 2 & 8 & 7 & 4 & 11 & 2.4 \\
0.64 & 14 & 1 & 7 & 7 & 4 & 9 & 2.0 \\
0.78 & 12 & 2 & 8 & 9 & 4 & 11 & 2.4 \\
0.82 & 14 & & & & & & \\
\hline
\end{tabular}


el momento que las plantas de primavera se irrigaron con las $\mathrm{SN}$, las plantas para el verano recibieron una $\mathrm{SN}$ con la fórmula de Steiner. Al presentar un diámetro de una pulgada en frutos del sexto racimo, correspondientes a frutos de verano, se dejó de aplicar la solución Steiner y se cambió por las SN con el respectivo balance K:Ca. Los riegos se efectuaron de acuerdo a las necesidades del cultivo aplicando un volumen de solución para mantener un $25 \%$ de drenaje.

Se registró el rendimiento por racimo y el rendimiento total acumulado se calculó con la suma de los cinco racimos de ambas estaciones. Se realizó un muestreo en el racimo 5 y en el racimo 10 , considerando un tamaño uniforme y color rojo, para determinar el contenido de sólidos solubles, firmeza y parámetros de color L, a y b.

Al término de cada estación se midió la altura de las plantas desde la base del tallo hasta la parte apical, mientras que el peso fresco de hojas, tallos y raíz se determinaron con una balanza digital. En frutos se determinó los parámetros de color mediante el software Tomato Analyzer versión 1.2. La firmeza de fruto se determinó con un penetrómetro (FT 327, McCormick ${ }^{\circledR}$ ) utilizando una puntilla de $8 \mathrm{~mm}$ de diámetro. Los datos climáticos prevalecientes en ambas estaciones del año se registraron mediante un Data Logger (Watch Dog series).

Cada uno de los balances K:Ca contó con cuatro repeticiones constituidas por dos plantas por repetición. El diseño experimental fue en bloques al azar y el análisis de datos se realizó mediante el software SAS versión 9.1 para correr un análisis de varianza; en casos de significancia se aplicó una prueba de medias por el método de Duncan $(P<0.05)$. Se relacionó el rendimiento de fruto por racimos de cada uno de los balances $\mathrm{K}: \mathrm{Ca}$ con el DPV y la RFA prevalecientes dentro del invernadero en cada una de las dos estaciones de evaluación.

\section{RESULTADOS Y DISCUSIÓN}

\section{Datos Ambientales Durante el Experimento}

Con los registros de temperatura (Figura 1) y humedad relativa (Figura 2) prevalecientes durante el experimento, se calculó el DPV (Figura 3) considerando este la diferencia entre la humedad absoluta a saturación a una temperatura máxima determinada y la humedad absoluta real a esa temperatura. La RFA se presenta en la Figura 4.
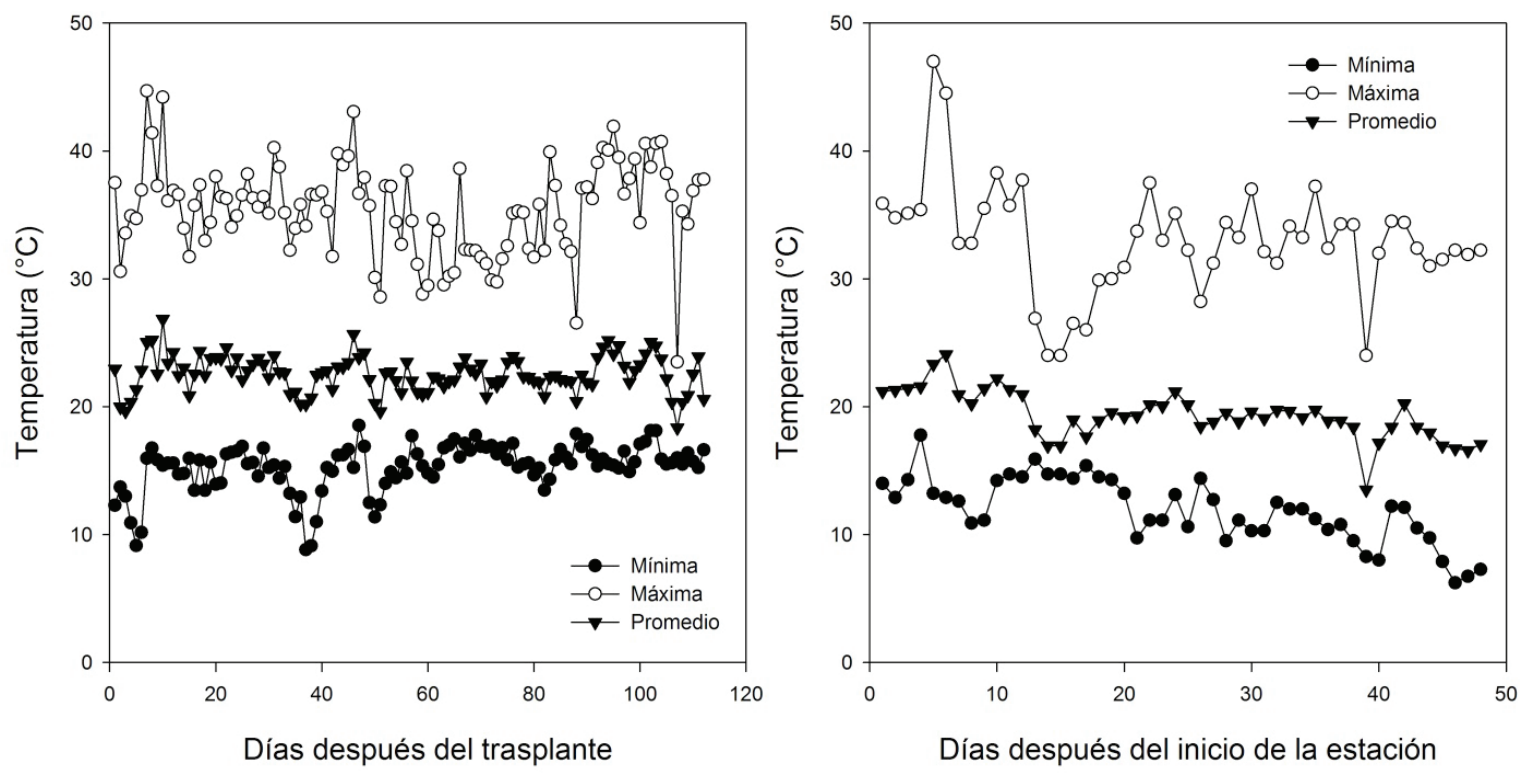

Figura 1. Temperatura mínima, máxima y promedio registrada en el invernadero durante el desarrollo de los frutos del primer a quinto racimo en la estación de primavera (izquierda) y del sexto al décimo racimo en la estación de verano (derecha).

Figure 1. Minimum, maximum and average temperature recorded in the greenhouse during the development of the fruits from the first to the fifth cluster in the spring season (left) and from the sixth to the tenth cluster in the summer season (right). 

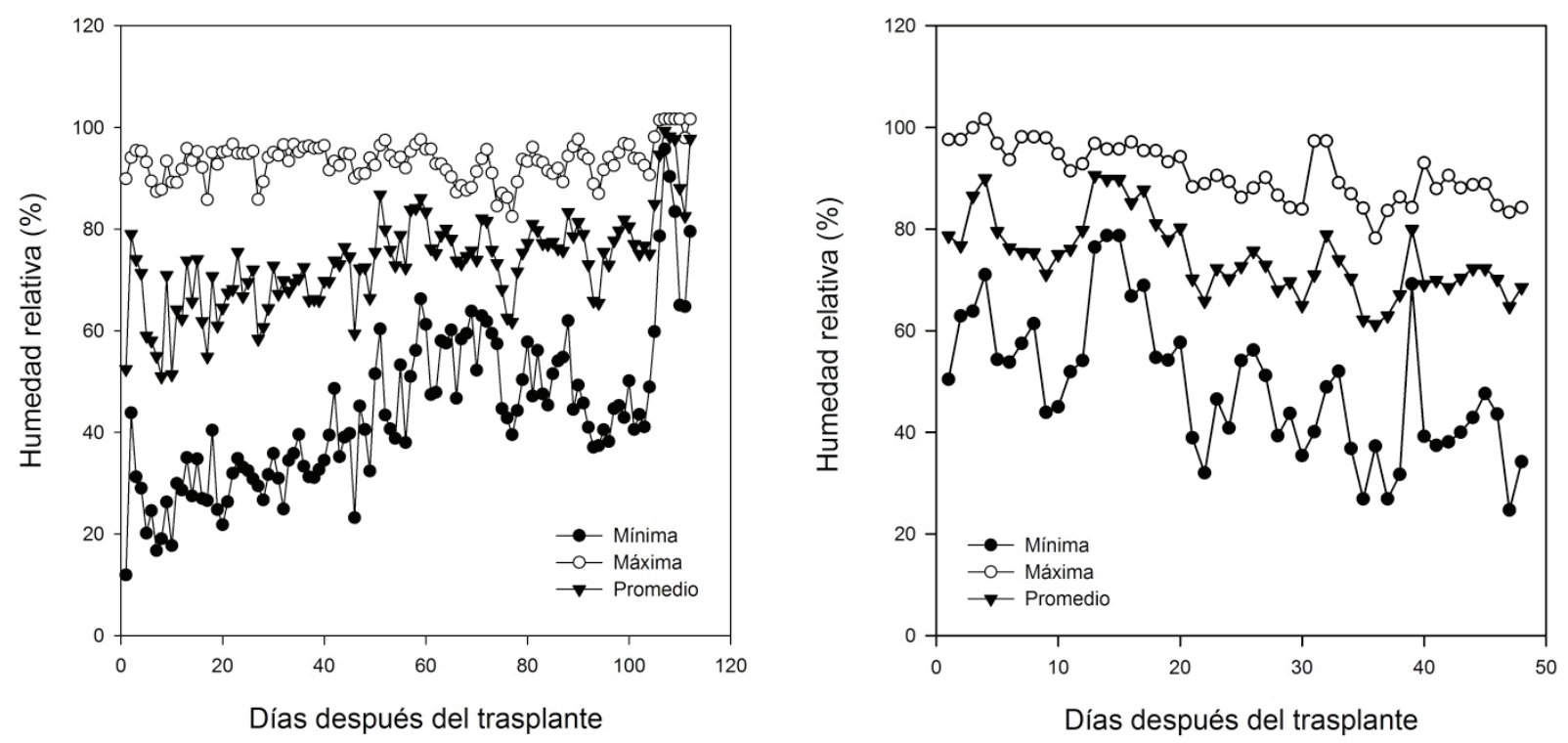

Figura 2. Humedad relativa mínima, máxima y promedio registradas en el invernadero durante el desarrollo de los frutos del primer a quinto racimo en la estación de primavera (izquierda) y del sexto al décimo racimo en la estación de verano (derecha).

Figure 2. Minimum, maximum and average relative humidity recorded in the greenhouse during the development of the fruits of the first to fifth cluster in the spring season (left) and from the sixth to the tenth cluster in the summer season (right).
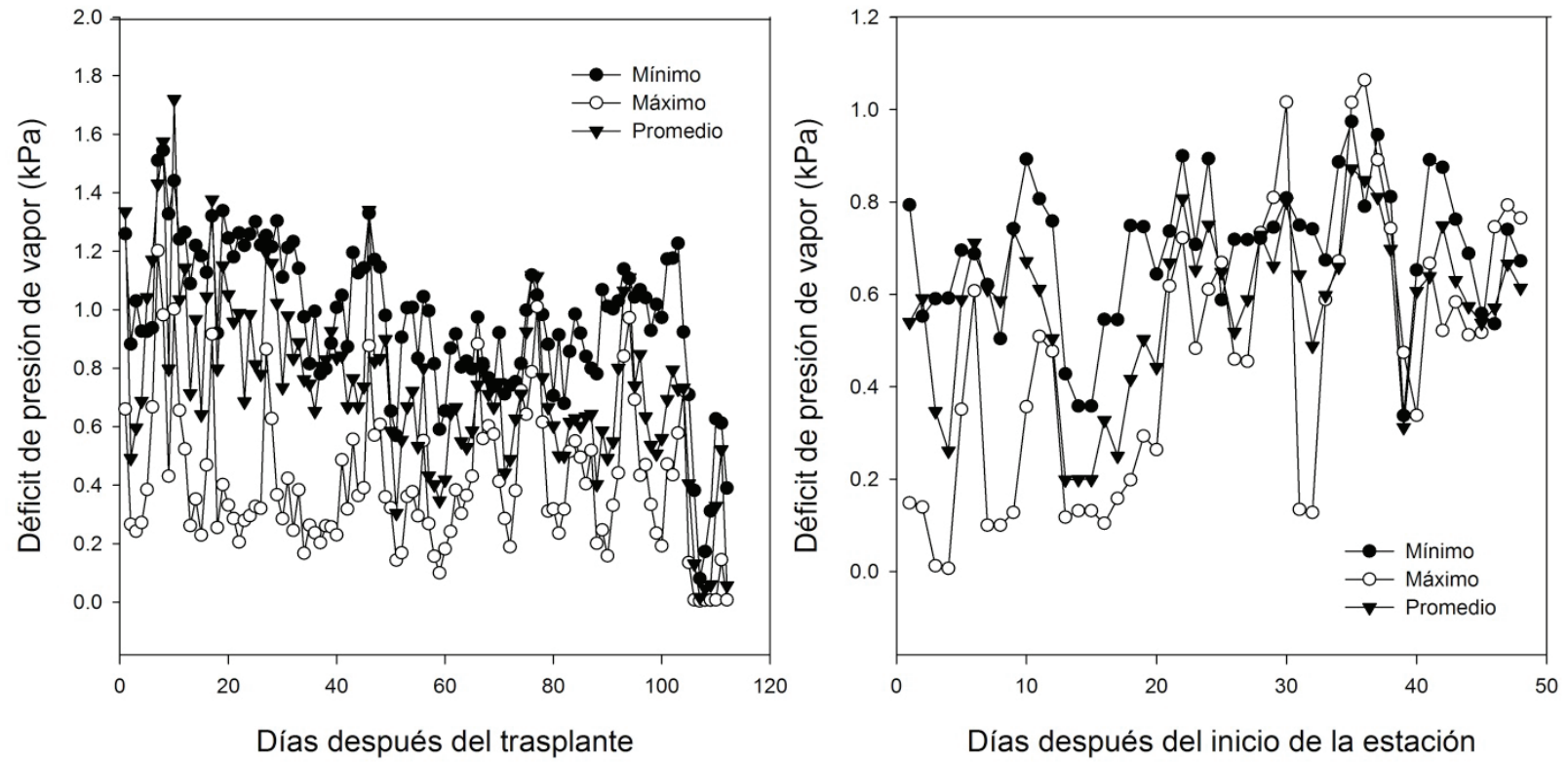

Figura 3. Déficit de presión de vapor registrado en el invernadero durante el desarrollo de los frutos del primer a quinto racimo en la estación de primavera (izquierda) y del sexto al décimo racimo en la estación de verano (derecha).

Figure 3. Vapor pressure deficit registered in the greenhouse during the development of the fruits from the first to the fifth cluster in the spring season (left) and from the sixth to the tenth cluster in the summer season (right). 

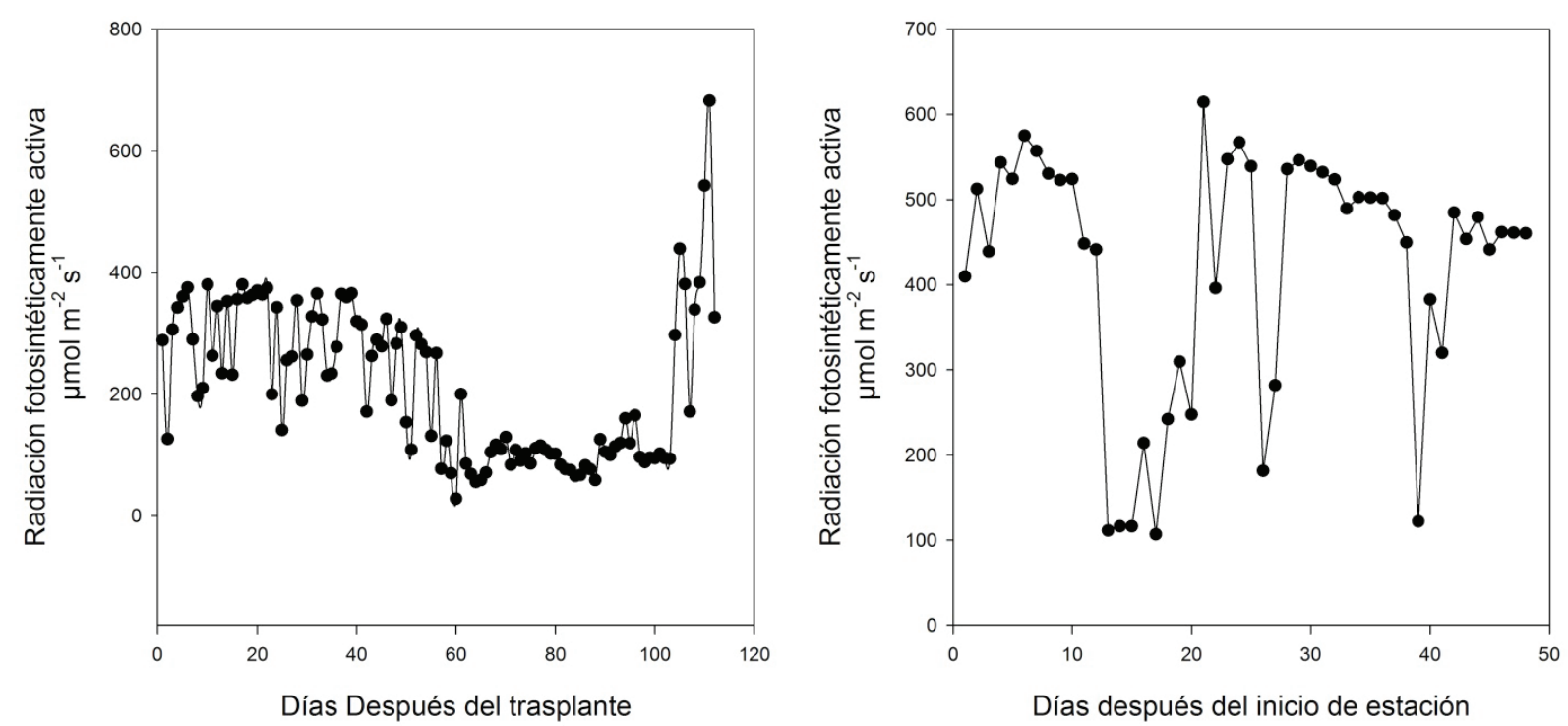

Figura 4. Radiación fotosintéticamente activa promedio registradas en el invernadero durante el desarrollo de los frutos del primer a quinto racimo en la estación de primavera (izquierda) y del sexto al décimo racimo en la estación de verano (derecha).

Figure 4. Average photosynthetically active radiation recorded in the greenhouse during the development of the fruits from the first to the fifth cluster in the spring season (left) and from the sixth to the tenth cluster in the summer season (right).

\section{Rendimiento y Calidad de Frutos}

En la cosecha de primavera, el balance K:Ca afectó el rendimiento acumulado del primero al quinto racimo (Cuadro 2), siendo las plantas tratadas con el balance de 0.64 las que obtuvieron el mayor rendimiento de fruto. En contraste, durante el verano, el rendimiento acumulado del sexto al décimo racimo no fue afectado significativamente (Cuadro 3).
A pesar de la ausencia de efecto en el rendimiento total acumulado durante la estación del año, el rendimiento de cada racimo, considerado individualmente, sí fue afectado por el balance $\mathrm{K}: \mathrm{Ca}$ tanto en la primavera como en el verano. En primavera, el balance que permitió el mayor rendimiento fue el de 0.64 para el cuarto y quinto racimo, mientras que el segundo racimo presentó mayor rendimiento con un balance de 0.78 (Figura 5). En cuanto a las cosechas

Cuadro 2. Efecto del balance K:Ca en la solución nutritiva aplicada durante el desarrollo y cosecha del primero al quinto racimo de frutos de tomate durante la estación de primavera.

Table 2. Effect of the K:Ca balance in the nutrient solution applied during the development and harvest of the first to the fifth cluster of tomato fruits during the spring season.

\begin{tabular}{|c|c|c|c|c|c|c|}
\hline Balance $\mathrm{K}: \mathrm{Ca}$ & $\begin{array}{l}\text { Rendimiento } \\
\text { frutos total }\end{array}$ & Altura de planta & $\begin{array}{l}\text { Peso fresco de } \\
\text { raíz }\end{array}$ & $\begin{array}{l}\text { Peso fresco de } \\
\text { tallo }\end{array}$ & $\begin{array}{c}\text { Peso fresco de } \\
\text { hojas }\end{array}$ & $\begin{array}{c}\text { Peso fresco } \\
\text { total }\end{array}$ \\
\hline & $\mathrm{kg}$ & $\mathrm{cm}$ & $\cdots \cdots$ & $--\mathrm{g}---$ & $-\cdots$ & \\
\hline 0.54 & $3.58 \mathrm{~b}^{\dagger}$ & 182 & 31.2 & $559 \mathrm{ab}$ & 1443 & $2034 \mathrm{ab}$ \\
\hline 0.64 & $3.88 \mathrm{a}$ & 179 & 49.5 & 578 a & 1612 & 2239 a \\
\hline 0.78 & $3.37 \mathrm{~b}$ & 187 & 32.4 & $505 \mathrm{bc}$ & 1197 & $1735 \mathrm{~b}$ \\
\hline 0.82 & $3.62 \mathrm{~b}$ & 183 & 40.3 & $488 \mathrm{c}$ & 1192 & $1719 \mathrm{~b}$ \\
\hline ANDEVA & $\mathrm{P}<0.01$ & $P=0.72$ & $P=16$ & $\mathrm{P}<0.02$ & $\mathrm{P}=0.07$ & $\mathrm{P}<0.04$ \\
\hline
\end{tabular}

${ }^{\dagger}$ Medias con letras diferentes en las columnas son estadísticamente diferente de acuerdo a la prueba Duncan con $P \leq 0.05$. ANDEVA $=$ análisis de varianza.

${ }^{\dagger}$ Averages with different letters in the columns are statistically different according to the Duncan test with $P \leq 0.05$. ANDEVA $=$ analysis of variance. 
Cuadro 3. Efecto del balance K:Ca en la solución nutritiva aplicada durante el desarrollo y cosecha del sexto al décimo racimo de frutos de tomate durante la estación de verano.

Table 3. Effect of the K:Ca balance in the nutrient solution applied during the development and harvest of the sixth to the tenth bunch of tomato fruits during the summer season.

\begin{tabular}{|c|c|c|c|c|c|c|}
\hline Balance $\mathrm{K}: \mathrm{Ca}$ & $\begin{array}{l}\text { Rendimiento } \\
\text { frutos total }\end{array}$ & Altura de planta & $\begin{array}{c}\text { Peso fresco de } \\
\text { raíz }\end{array}$ & $\begin{array}{l}\text { Peso fresco de } \\
\text { tallo }\end{array}$ & $\begin{array}{c}\text { Peso fresco de } \\
\text { hojas }\end{array}$ & $\begin{array}{c}\text { Peso fresco } \\
\text { total }\end{array}$ \\
\hline & $\mathrm{kg}$ & $\mathrm{cm}$ & $-\cdots$ & $---g--$ & $-\cdots$ & \\
\hline 0.54 & 2.77 & 295 & 40.1 & 540 & 1686 & 2266 \\
\hline 0.64 & 2.51 & 279 & 46.4 & 519 & 1551 & 2117 \\
\hline 0.78 & 2.86 & 286 & 38.1 & 562 & 1598 & 2198 \\
\hline 0.82 & 2.78 & 283 & 42.2 & 529 & 1733 & 2304 \\
\hline ANDEVA & $\mathrm{P}=0.32$ & $\mathrm{P}=0.40$ & $\mathrm{P}=0.39$ & $P=0.76$ & $P=0.67$ & $P=0.77$ \\
\hline
\end{tabular}

ANDEVA = análisis de varianza. ANDEVA = analysis of variance.

realizadas en verano, el rendimiento fue mayor en el sexto, séptimo y noveno racimos cuando el balance K: Ca fue de 0.54, 0.78 y 0.78, respectivamente, mientras que en el octavo racimo el rendimiento sólo fue afectado negativamente con el balance de 0.64 (Figura 6).

Lo anterior sugiere que las condiciones ambientales prevalecientes durante el desarrollo de cada uno de los racimos afectaron el rendimiento de fruto. Se ha reportado que la polinización es sensible a temperaturas altas, como las observadas en la primavera (Figura 1), por lo que el rendimiento se vería afectado (Hatfield y Prueger, 2015); asimismo, se ha reportado que

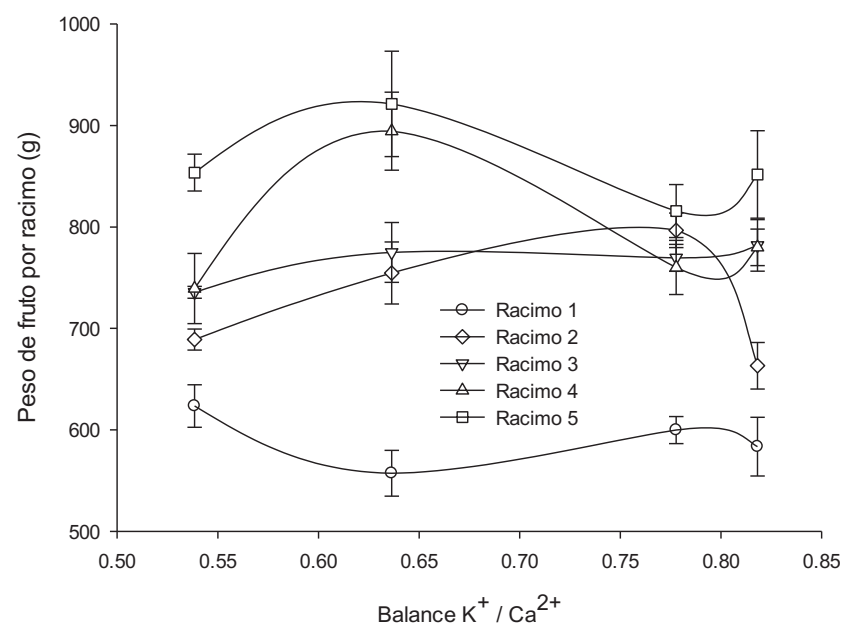

Figura 5. Efecto de balance K:Ca de la solución nutritiva sobre el rendimiento del primero al quinto racimo de frutos de tomate desarrollados y cosechados durante la primavera.

Figure 5. K:Ca balance effect of the nutrient solution on the yield of the first to the fifth cluster of tomato fruits developed and harvested during the spring. un aumento del 50 al $75 \%$ en la HR incrementa la viabilidad del polen (Harel et al., 2014), lo que favorece el rendimiento de frutos. Un incremento de $2.5^{\circ} \mathrm{C}$ por encima de la temperatura óptima para tomate de $25^{\circ} \mathrm{C}$ es suficiente para reducir el rendimiento hasta en un 40\% (Harel et al., 2014).

En el presente estudio, el DPV calculado durante el desarrollo de los frutos en el respectivo racimo tuvo una relación directa con el rendimiento; en la Figura 7 se observa que durante la primavera, el rendimiento de fruto en cada uno de los cinco racimos estuvo influenciado por el balance K:Ca así como por el DPV.

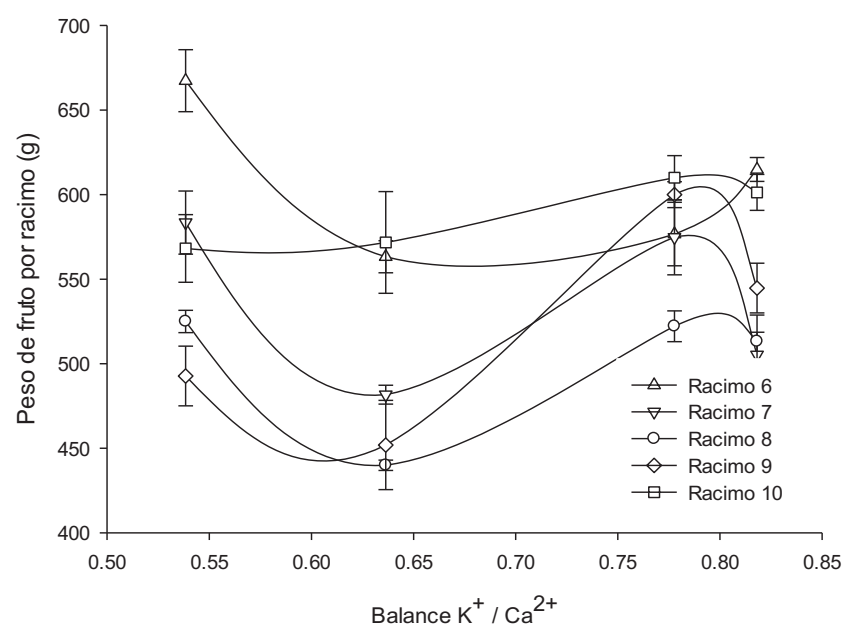

Figura 6. Efecto de balance K:Ca de la solución nutritiva sobre el rendimiento del sexto al décimo racimo de frutos de tomate desarrollados y cosechados durante el verano.

Figure 6. K:Ca balance effect of the nutrient solution on the yield of the sixth to the tenth cluster of tomato fruits developed and harvested during the summer. 


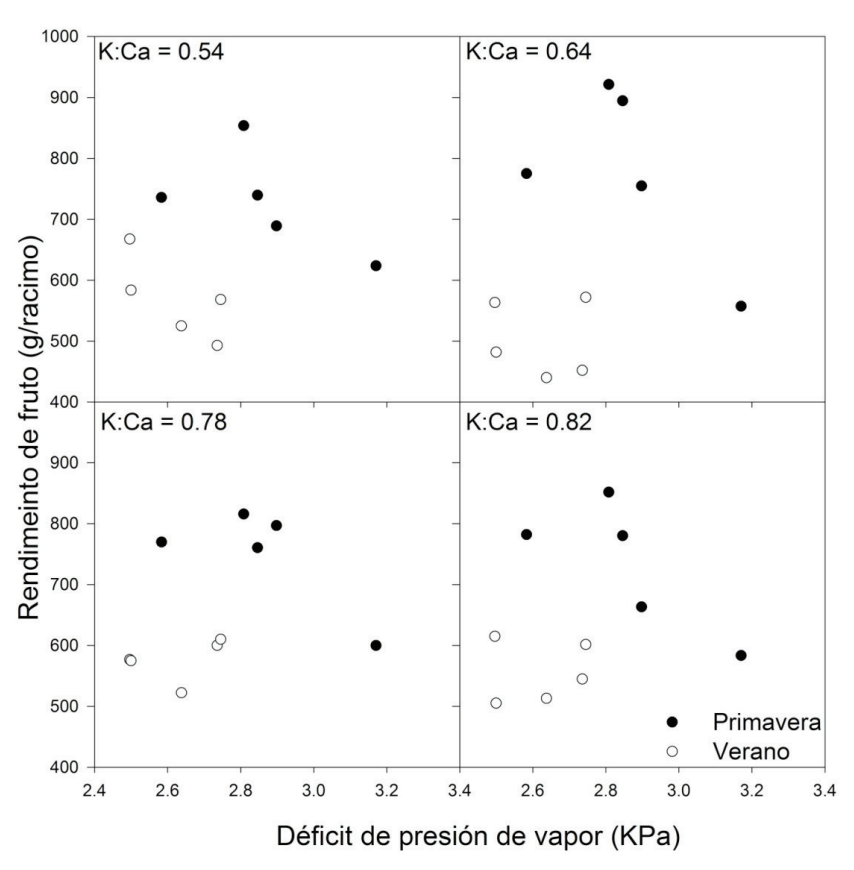

Figura 7. Efecto de déficit de presión de vapor en dos estaciones del año sobre el rendimiento de fruto de tomate en función del balance K:Ca en la solución nutritiva.

Figure 7. Effect of vapor pressure deficit in two seasons of the year on tomato fruit yield as a function of the $\mathrm{K}$ : Ca balance in the nutrient solution.

Los racimos tuvieron un mayor rendimiento de fruto cuando el balance K:Ca fue de 0.64 y el DPV fue de 2.81 $\mathrm{KPa}$. En contraste, durante el verano, el rendimiento más alto se obtuvo en frutos de plantas tratadas con un balance de 0.54 y el DPV fue 2.50 o $2.74 \mathrm{KPa}$.

El hecho de que en primavera se requiera de un balance $\mathrm{K}: \mathrm{Ca}$ más alto $(\mathrm{K}: \mathrm{Ca}=0.64)$ que en verano $(\mathrm{K}: \mathrm{Ca}=0.54)$ implica que en el primer caso las plantas demandan menos $\mathrm{Ca}$ (Cuadro 1) mientras que lo opuesto ocurre en verano, cuando se presenta mayor demanda de $\mathrm{Ca}$ (Cuadro 1). A su vez, el balance más alto en primavera puede estar relacionado con la demanda evaporativa por parte de las plantas, ya que el DPV óptimo fue más elevado en primavera que en el verano.

Se ha reportado que la tasa de acumulación de $\mathrm{Ca}$ se encuentra estrechamente ligada a la transpiración (Airman y Houter, 1990), y esta última al DPV. Un mayor DPV implica que el aire del invernadero está menos saturado de humedad, y por lo tanto la tasa de transpiración puede ser más elevada, lo que permite un mayor transporte y movilización el Ca a través del xilema a pesar de estar en menor proporción en relación al K (Hawkesford et al., 2012).

Por el contrario, en el verano, el DPV óptimo fue más bajo que en primavera, lo que implica que el aire se encuentra más saturado de humedad, favoreciendo una tasa de transpiración más baja y obligando de esta manera a elevar la proporción de Ca en la $\mathrm{SN}$ pues este se translocaría también a una menor velocidad. Estos resultados coinciden con lo reportado por del Amor y Marcelis (2006), quienes señalan que una alta HR reduce la concentración de $\mathrm{Ca}$ en las partes vegetativas de plantas de tomate. Leonardi et al. (2000) reportan que un alto DPV $(2.2 \mathrm{KPa})$ ocasiona una reducción en el peso fresco de frutos, pero con un incremento en los sólidos solubles en comparación con plantas cultivadas en ambientes con un menor DPV (1.6 KPa); lo anterior está en concordancia con nuestros resultados pues el rendimiento de frutos fue más bajo en verano (con un mayor DPV), que en primavera (con un mayor DPV).

ElbalanceK:Ca que permitió el rendimiento más alto en cada uno de los cinco racimos desarrollados durante la primavera estuvo correlacionado negativamente con el DPV (Figura 8). En contraste, la correlación entre el balance $\mathrm{K}$ :Ca óptimo durante el verano fue positiva. Lo anterior sugiere que una estrategia para contrarrestar el efecto negativo de las condiciones ambientales del invernadero prevalecientes durante una determinada estación del año es el ajuste del balance $\mathrm{K}: \mathrm{Ca}$ de las soluciones nutritivas en función del DPV y de la estación del año, pues mientras que en primavera se recomienda un menor balance $\mathrm{K}: \mathrm{Ca}$ bajo condiciones de un alto DPV, en verano un alto DPV debe ir acompañado de un balance K:Ca más elevado.

La diferencia en la respuesta al DPV entre ambas estaciones puede ser debido a la interacción con la RFA, ya que esta también tuvo un efecto definido sobre el balance $\mathrm{K}$ :Ca que produjo el rendimiento más elevado en cada uno de los cinco racimos producidos en cada estación del año. En primavera, se observó que el balance K:Ca óptimo debe ser de 0.78-0.82 al subir la RFA hasta un máximo de $102-142 \mu \mathrm{mol} \mathrm{m}^{-2} \mathrm{~s}^{-1}$, mientras que niveles más bajos o altos deben ir acompañados de una reducción en el balance K:Ca (Figura 8). Una tendencia similar fue observada durante el verano, pues valores extremos de RFA deben ir acompañadas de un balance $\mathrm{K}: \mathrm{Ca}$ de 0.55 , mientras bajo una RFA de $479-475 \mu \mathrm{mol} \mathrm{m}{ }^{-2} \mathrm{~s}^{-1}$ el balance debe ser de 0.78 (Figura 8). 

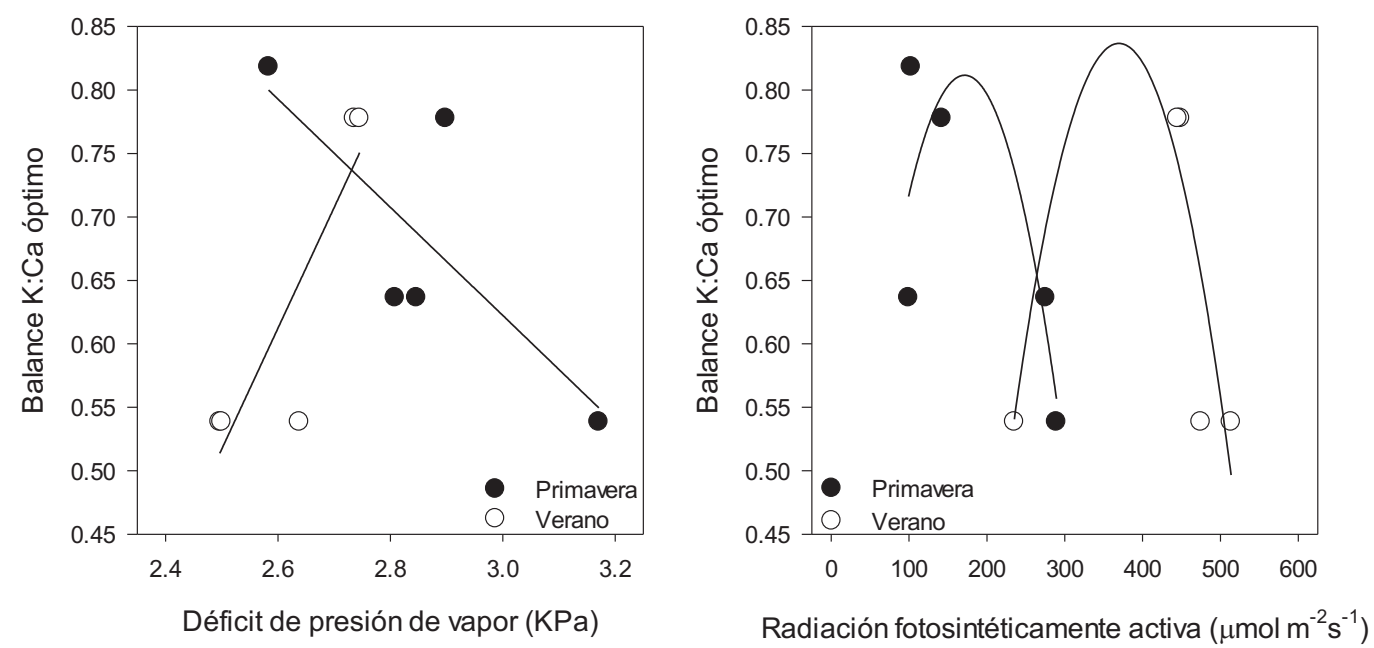

Figura 8. Relación entre el déficit de presión de vapor y radiación fotosintéticamente activa sobre el balance K:Ca bajo los cuales se obtuvieron los rendimientos más altos en cada uno de los cinco racimos evaluados en dos estaciones del año.

Figure 8. Relationship between vapor pressure deficit and photosynthetically active radiation on the $\mathrm{K}$ :Ca balance, under which the highest yields were obtained in each of the five clusters evaluated in two seasons of the year.

Considerando los datos obtenidos durante ambas estaciones de cultivo, el modelo que explica el efecto del DPV y la RFA así como la interacción entre ambos factores sobre el rendimiento es:

Rendimiento de fruto $(\mathrm{kg} / \mathrm{planta})=247.3+339.6 \mathrm{DPV}$ +11.1 RFA - 3.77 (DPV $\times$ RFA), $\mathrm{R}^{2}=0.763, \mathrm{P}<0.001$

mientras que el modelo que explica el efecto del balance $\mathrm{K}$ :Ca en combinación con el DPV y RFA es:

Rendimiento de fruto $(\mathrm{kg} / \mathrm{planta})=588.9+107.6$ $(\mathrm{K}: \mathrm{Ca} \times \mathrm{DPV})-0.038(\mathrm{~K}: \mathrm{Ca} \times \mathrm{RFA} \times \mathrm{DPV}), \mathrm{R}^{2}=$ $0.416, \mathrm{P}<0.001$

Leonardi et al. (2000) reportaron que el color del fruto de tomate no es afectado por el DPV; estos resultados coinciden con los observados en el presente estudio puesto que, además del color, otros parámetros de calidad como la firmeza no fueron afectados por el DPV prevaleciente en ninguna de las estaciones de cultivo ni por el balance K:Ca en la SN (Cuadros 4 y 5). Sin embargo, la concentración de sólidos solubles en los frutos cosechados en el verano fue mayor al de los cosechados en primavera, aunque estos fueron reducidos significativamente cuando las plantas se nutrieron con soluciones conteniendo un balance $\mathrm{K}: \mathrm{Ca}$ de 0.64 (Cuadro 4). Resultados similares han sido reportados por Leonardi et al. (2000) ya que con un alto DPV se produjo un incremento en los sólidos solubles en comparación con plantas crecidas en ambientes con un menor DPV.

\section{Respuestas Agronómicas}

El peso fresco de tallo y total fueron más altos en plantas desarrolladas durante la primavera y irrigadas con soluciones con un balance $\mathrm{K}: \mathrm{Ca}$ de 0.64 (Cuadro 2). Durante el verano, no se detectaron efectos significativos sobre la biomasa de las plantas (Cuadro 3). El mayor peso fresco total conforme se aumentó la proporción de $\mathrm{Ca}$ en relación a la de $\mathrm{K}$ puede explicarse por el efecto de este nutrimento en el estado hídrico de la plantas, lo que resultaría en un mayor contenido de agua (Peil y Galvez, 2005); éstos resultados son similares a los reportados por Shabala y Pottosin (2014) y Shabani et al. (2012), quienes señalaron que concentraciones crecientes de $\mathrm{K}$ en la SN sobre tomate tipo cereza bajo condiciones salinas aumentó el crecimiento de las plantas. 
Cuadro 4. Efecto del balance K:Ca en la solución nutritiva sobre la calidad de frutos de tomate durante el desarrollo y cosecha del primero al quinto racimo de frutos en la primavera.

Table 4. Effect of the K:Ca balance in the nutrient solution on the quality of tomato fruits during the development and harvest of the first to the fifth cluster of fruits in the spring.

\begin{tabular}{lccccc}
\hline Balance K:Ca & Firmeza & Sólidos solubles totales & $* \mathrm{~L}$ & $*_{\mathrm{a}}$ & $* \mathrm{~b}$ \\
\hline \multirow{2}{*}{0.54} & $\mathrm{~kg} \mathrm{~cm}^{-2}$ & ${ }^{\circ}$ Brix & & & \\
0.64 & 2.97 & 5.0 & 36.8 & 42.6 & 43.9 \\
0.78 & 2.49 & 5.1 & 37.3 & 41.9 & 44.6 \\
0.82 & 2.77 & 5.0 & 36.7 & 42.2 & 44.7 \\
ANDEVA & 2.43 & 5.3 & 36.7 & 43.2 & 44.2 \\
& $\mathrm{P}=0.53$ & $\mathrm{P}=0.29$ & $\mathrm{P}=0.45$ & $\mathrm{P}=0.78$ & $\mathrm{P}=0.66$ \\
\hline
\end{tabular}

ANDEVA = análisis de varianza. ANDEVA = analysis of variance.

Cuadro 5. Efecto del balance K:Ca en la solución nutritiva sobre la calidad de frutos de tomate durante el desarrollo y cosecha del primero al quinto racimo de frutos en el verano.

Table 5. Effect of the K:Ca balance in the nutrient solution on the quality of tomato fruits during the development and harvest of the first to the fifth cluster of fruits in the summer.

\begin{tabular}{|c|c|c|c|c|c|}
\hline Balance $\mathrm{K}: \mathrm{Ca}$ & Firmeza & Sólidos solubles totales & $* \mathrm{~L}$ & $*_{\mathrm{a}}$ & $* \mathrm{~b}$ \\
\hline & $\mathrm{kg} \mathrm{cm}^{-2}$ & ${ }^{\circ}$ Brix & & & \\
\hline 0.54 & 2.95 & $6.3 \mathrm{~b}$ & 35.1 & 39.4 & 40.6 \\
\hline 0.64 & 2.89 & $5.6 \mathrm{c}$ & 35.2 & 39.9 & 41.3 \\
\hline 0.78 & 2.88 & $6.0 \mathrm{bc}$ & 35.1 & 39.9 & 41.0 \\
\hline 0.82 & 3.10 & $6.6 \mathrm{a}$ & 35.0 & 39.8 & 40.4 \\
\hline ANDEVA & $\mathrm{P}=0.83$ & $\mathrm{P}=0.02$ & $\mathrm{P}=0.97$ & $P=0.96$ & $\mathrm{P}=0.49$ \\
\hline
\end{tabular}

ANDEVA $=$ análisis de varianza. ANDEVA = analysis of variance.

\section{CONCLUSIONES}

El balance K:Ca óptimo mejoró el rendimiento, pero este es afectado por las condiciones ambientales en las cuales se desarrolla cada racimo así como por la estación del año. El rendimiento responde positivamente a un balance $\mathrm{K}$ : Ca de 0.64 combinado con un DPV de $2.81 \mathrm{KPa}$ en primavera, mientras que en verano el balance óptimo fue el de 0.54 combinado con un DPV de 2.50 o $2.74 \mathrm{KPa}$. En primavera, un incremento en el DPV debe ir acompañado de una reducción en el balance $\mathrm{K}: \mathrm{Ca}$, mientras que en el verano debe ser lo opuesto. Con excepción de los sólidos solubles totales, la calidad de los frutos, así como la biomasa, no fueron afectados significativamente. En primavera, el balance $\mathrm{K}$ :Ca óptimo debe ser de 0.78-0.82 al subir la RFA hasta un máximo de $102-142 \mu \mathrm{mol} \mathrm{m} \mathrm{m}^{-2} \mathrm{~s}^{-1}$, mientras que niveles más bajos o altos deben ir acompañados de una reducción en el balance $\mathrm{K}: \mathrm{Ca}$, mientras que en el verano, valores extremos de RFA deben ir acompañadas de un balance $\mathrm{K}: \mathrm{Ca}$ de 0.55 , mientras bajo una RFA de $479-475 \mu \mathrm{mol} \mathrm{m}^{-2} \mathrm{~s}^{-1}$ el balance debe ser de 0.78 .

\section{LITERATURA CITADA}

Adams, P. and L. C. Ho. 1995. Uptake and distribution of nutrients in relation to tomato fruit quality. Acta Hortic. 412: 374-387. doi: https://doi.org/10.17660/ActaHortic.1995.412.45.

Airman, D. P. and G. Houter. 1990. Influence of radiation and humidity on transpiration: Implications for calcium levels in tomato leaves. J. Hortic. Sci. 65: 245-253. doi: https://doi.org/ 10.1080/00221589.1990.11516053.

Almeselmani, M., R. C. Pant, and B. Singh. 2009. Potassium level and physiological response and fruit quality in hydroponically grown tomato. Int. J. Veget. Sci. 16: 85-99. doi: 10.1080/19315260903271526. 
Amor, F. M. and L. F. M. Marcelis. 2006. Differential effect of transpiration and $\mathrm{Ca}$ supply on growth and $\mathrm{Ca}$ concentration of tomato plants. Sci. Hortic. 111: 17-23. doi: https://doi. org/10.1016/j.scienta.2006.07.032.

Barrón, M. A. and F. Rello. 2000. The impact of the tomato agroindustry on the rural poor in México. Agric. Econ. 23: 289297. doi: https://doi.org/10.1016/S0169-5150(00)00090-6.

Bertin, N., S. Guichard, C. Leonardi, J. J. Longuenesse, D. Langlois, and B. Navez. 2000. Seasonal evolution of the quality of fresh glasshouse tomatoes under Mediterranean conditions, as affected by air vapor pressure deficit and plant fruit load. Ann. Bot: 85: 741-750. doi: https://doi.org/10.1006/ anbo.2000.1123.

Betancourt, P. y F. Pierre. 2013. Extracción de macronutrientes por el cultivo de tomate (Solanum lycopersicum Mill. var. Alba) en casas de cultivo en Quíbor, Estado Lara. Bioagro 25: 181-188.

Díaz, A., G. Cayón y J. J. Mira. 2007. Metabolismo del calcio y su relación con la "mancha de madurez" del fruto de banano. Una revisión. Agron. Colomb. 25: 280-287.

Fanasca, S., G. Colla, G. Maiani, E. Venneria, Y. Rouphael, E. Azzini, E., and F. Saccardo. 2006. Changes in antioxidant content of tomato fruits in response to cultivar and nutrient solution composition. J. Agric. Food Chem. 54: 4319-4325. doi:10.1021/jf0602572.

Harel, D., H. Fadida, A. Slepoy, S. Gantz, and K. Shilo. 2014. The effect of mean daily temperature and relative humidity on pollen, fruit set and yield of tomato grown in commercial protected cultivation. Agronomy 4: 167-177. doi: 10.3390/ agronomy4010167.

Hatfield, J. L. and J. H. Prueger. 2015. Temperature extremes : Effect on plant growth and development. Weather Clim. Extr. 10: 4-10. doi: https://doi.org/10.1016/j.wace.2015.08.001.

Hawkesford, M., W. Horst, T. M. R. Kichey, J. K. Schjørring, H. Lambers, I. S. Møller, and P. White. 2012. Functions of macronutrients. pp. 135-189. In: P. Marschner (ed.). Marschner's mineral nutrition of higher plants. Academic Press. New York, NY, USA. doi: https://doi.org/10.1016/ B978-0-12-384905-2.00006-6.

Lara H., A. 1999. Manejo de la solución nutritiva en la producción de tomate en hidroponía. Terra 17: 221-229.

Leonardi, C., S. Guichard, and N. Bertin. 2000. High vapour pressure deficit influences growth, transpiration and quality of tomato fruits. Sci. Hortic. 84: 285-296. doi: https://doi. org/10.1016/S0304-4238(99)00127-2.

Nukaya, A., K. Goto, H. Jang, A. Kano, and K. Ohkawa. 1995. Effect of $\mathrm{K} / \mathrm{Ca}$ ratio in the nutrient solution on incidence of blossom-end rot and gold specks of tomato of tomato fruit grown in rockwool. Acta Hortic. 396: 123-130. doi: 10.17660/ ActaHortic.1995.396.13.

Paiva, E. A. S., R. Arruda Sampaio, and H. E. Prieto Martinez. 1998. Composition and quality of tomato fruit cultivated in nutrient solutions containing different calcium concentrations. J. Plant Nutr. 21: 2653-2661. doi: https://doi. org/10.1080/01904169809365595.
Parra T., S. P., M .Villarreal R., P. Sánchez P., J. L. Corrales Madrid y S. Hernández V. 2008. Efecto del calcio y potencial osmótico de la solución nutritiva en la pudrición apical, composición mineral y rendimiento de tomate. Interciencia 33: 449-456.

Peil, R. M. y J. L. Galvez. 2005. Reparto de materia seca como factor determinante de la producción de las hortalizas de fruto cultivadas en invernadero. Rev. Bras. Agroc. 11: 5-11. doi: HTTP://DX.DOI.ORG/10.18539/CAST.V11I1.1171.

Riga, P., M. Anza, and C. Garbisu. 2008. Tomato quality is more dependent on temperature than on photosynthetically active radiation. J. Sci. Food Agric. 88: 158-166. doi: https://doi. org/10.1002/jsfa.3065.

Saavedra, T. M., G. A. Figueroa, and J. G. D. Cauih. 2017. Origin and evolution of tomato production Lycopersicon esculentum in México. Ciênc. Rural 47: e20160526. doi: https://doi. org/10.1590/0103-8478cr20160526.

Schneider, A. and A. Mollier. 2016. Modelling of K/Ca exchange in agricultural soils. Geoderma 271: 216-224. doi: 10.1016/j. geoderma.2016.02.016.

Shabala, S. and I. Pottosin. 2014. Regulation of potassium transport in plants under hostile conditions: Implications for abiotic and biotic stress tolerance. Physiol. Plant. 151: 257-279. doi: 10.1111/ppl.12165.

Shabani, E., S. J. Tabatabaei, S. Bolandnazar, and K. Ghasemi. 2012: Vegetative growth and nutrient uptake of salinity stressed cherry tomato in different calcium and potassium level. Int. Res. J. Appl. Basic Sci. 3: 1845-1853.

SIAP (Servicio de Información Agroalimentaria y Pesquera). 2018. Atlas Agroalimentario 2018. Publicaciones SIAP. https://nube. siap.gob.mx/gobmx_publicaciones_siap/pag/2018/ (Consulta: julio 2, 2019).

Soto Hernández, D. 2018. Análisis de la competitividad del tomate Mexicano en el contexto del TLCAN: escenario ante el mercado Chino. Rev. Econ. Admin. 15: 15-31.

Suzuki, M., H. Umeda, S. Matsuo, Y. Kawasaki, D. Ahn, H. Hamamoto, and Y. Iwasaki. 2015. Effects of relative humidity and nutrient supply on growth and nutrient uptake in greenhouse tomato production. Sci. Hortic. 187: 44-49. doi: 10.1016/j.scienta.2015.02.035.

Swaef, T. and K. Steppe. 2010. Linking stem diameter variations to sap flow, turgor and water potential in tomato. Funct. Plant Biol. 37: 429-438. doi: 10.1071/FP09233.

Van den Broeck, G. and M. Maertens. 2016. Horticultural exports and food security in developing countries. Global Food Sec. 10: 11-20. doi: 10.1016/j.gfs.2016.07.007.

Villegas-Torres, O. G., P. Sánchez-García, G. A. Baca-Castillo, M. N. Rodríguez-Mendoza, C. Trejo, M. Sandoval-Villa y E. Cárdenas-Soriano. 2005. Crecimiento y estado nutrimental de plántulas de tomate en soluciones nutritivas con diferente concentración de calcio y potencial osmótico. Terra Latinoamericana 23: 49-56. 